\title{
Some Properties of Random Fixed Points and Random Periodic Points for Random Dynamical Systems
}

\author{
Sundus Talib Mohsin Ihsan Jabbar Kadhim \\ University of Al-Qadiysih, College of Computer Science and Information \\ Technology \\ sundustalibm@gmail.com \\ Ihsan.kadhim@qu.edu.iq
}

Recived : 17\7\2018

Revised : 11812018

Accepted : $1618 \backslash 2018$

Available online : $25 / 9 / 2018$

DOI: $10.29304 / j q c m .2018 .10 .3 .432$

\begin{abstract}
Our aim in this paper is to give some properties for random fixed points for random dynamical systems where we give the characteristic of random fixed points in terms of the random trajectory emanating from random variable and give. Also, the concept of random periodic points for random dynamical systems is studied where the sufficiently and necessarily conditions which make the random variable random periodic point for random dynamical systems. Also the authors prove that the set of all (continuous) random periodic points under certain conditions is $\mathbb{P}$-closed.
\end{abstract}

Key Words: Random dynamical system; random fixed point; random periodic point.

Mathematics subject classification:37HXX. 
1-Introduction. In common one can not anticipate that one point $x \in X$ is fixed by (almost) all mappings $\varphi(t, \omega)$. Though, there is a suitable generalization of the idea of a fixed point. In this paper some new properties of random fixed point and random periodic point for RDS's are considered.

L. Arnold and I.D. Chueshov [1] (1998) presented the general notion of an order-preserving random dynamical system, gave several examples and studied the properties of their random equilibria and attractor.

Gunter Ochs and Valery. Oseledets [2] (1999) establish that topological fixed point theorems have no canonical generalization to the case of random dynamical systems. This is prepared by exhausting implements from algebraic ergodic theory. They provide a condition for the existence of invariant probability measures for group valued cocycles. With that, examples of continuous random dynamical systems on a compact interval without random invariant points, which are an suitable generalization of fixed points, are created. H.E. Kunze D. La Torreb and E.R. Vrscay[3](2007) they absorbed in the direct and inverse problems for certain class of random fixed point equations.

Chuanxi Zhu and Chunfang Chen[4](2008),they prove an essential inequality and inspect some new computing problems of random fixed point index.

"Ismat Beg and Mujahid Abbas[5](2008) they prove the existence of random fixed points of a non-expansive random operator defined on an unbounded subset of a Banach space".
In this paper some new properties of random fixed points and periodic random points for random dynamical system are introduced and proved. Here the time space considered any locally compact space and the phase space is any metric space. Also some new concepts are introduced here such as Topological metric dynamical system, $\mathbb{P}-$ uniform converge and $\mathbb{P}$-closed set.

Through this paper the following notation are used.

\section{Notations 1.1}

(i) $\mathbb{G}=$ locally compact group.

(ii) $\mathbb{X}=$ metric space.

(iii) $(\Omega, \mathbb{F}, \mathbb{P})$ is a probability space.

(iv) $\mathbb{X}_{\mathcal{B}}^{\Omega}=$ the set of all measurable functions from $\Omega$ to $\mathbb{X}$.

Definition 1.2[6,7]:The metric dynamical system $(\mathrm{MDS})$ is the 5-tuple $(\mathbb{G}, \Omega, \mathbb{F}, \mathbb{P}, \theta)$ where $(\Omega, \mathbb{F}, \mathbb{P})$ is a probability space and $\theta: G \times \Omega \rightarrow \Omega$ is $(\beta(\mathbb{G}) \otimes \mathbb{F}, \mathbb{F})$-measurable, with

(i) $\quad \theta(e, \omega)=I d_{\Omega}$, (the identity function on $\Omega$ )

(ii) $\quad \theta(g * h, \omega)=\theta(g, \theta(h, \omega))$ and

(iii) $\mathbb{P}\left(\theta_{g} F\right)=\mathbb{P}(F), \forall F \in \mathbb{F} \forall g \in G$.

Definition 1.3 The $\operatorname{MDS}(\mathbb{G}, \Omega, \mathbb{F}, \mathbb{P}, \theta)$ is said to be topological metric dynamical system (TMDS) if $\Omega$ is topological space and $\theta: G \times \Omega \rightarrow \Omega$ is continuous.

Definition 1.4[6,7,8] The mapping $\varphi: \mathbb{G} \times \Omega \times$ $\mathbb{X} \rightarrow \mathbb{X}$ is said to be measurable random dynamical system on the measurable space $(\mathbb{X}, \beta(\mathbb{X}))$ over an $\operatorname{MDS}(\mathbb{G}, \Omega, \mathbb{F}, \mathbb{P}, \theta)$ with if it has the following properties:

(i) $\varphi$ is $\beta(\mathbb{G}) \otimes \mathbb{F} \otimes \beta(\mathbb{X}), \beta(\mathbb{X})$ - measurable.

(ii) The mappings $\varphi(t, \omega):=\varphi(g, \omega, \cdot): \mathbb{X} \rightarrow \mathbb{X}$ form a cocycle over $\theta(\cdot)$, that is, $\forall g, h \in \mathbb{G}, \omega \in$ $\Omega$ they satisfy 
Sundus .T/Ihsan .J

$\varphi(e, \omega)=i d_{X} \forall \omega \in \Omega, \quad(1.1)$

$\varphi(g * h, \omega)=\varphi\left(g, \theta_{h} \omega\right) \circ \varphi(h, \omega)(1.2)$

The RDS $(\mathbb{G}, \Omega, \mathbb{X}, \theta, \varphi)$ shall denote by $(\theta, \varphi)$. If the function $\varphi(\cdot, \omega, \cdot): \mathbb{T} \times \mathbb{X} \rightarrow \mathbb{X},(t, x) \mapsto$ $\varphi(t, \omega, x)$, is continuous for every $\omega \in \Omega$ then the measurable dynamical system is called continuous or topological RDS.

Definition 1.5 [6,7,8]: A measurable function $v \in X_{\mathcal{B}}^{\Omega}$ is said to be a random fixed point (R.F.P) for the $\operatorname{RDS}(\theta, \varphi)$ if,$\forall g \in G$, $\mathbb{P}\left\{\omega: \varphi(g, \omega) v(\omega)=v\left(\theta_{g} \omega\right)\right\}=1$.

Here some examples on R.F.P are stated (see[9]).

Example 1.6[9] Consider the probability space $(\Omega, \mathcal{F}, \mathbb{P})$, where $\Omega:=[0,1], \mathcal{F}$ be the $\sigma-$ algebra of Lebesgue measurable sets and $\mathbb{P}$ be the Lebesgue measurer on $\Omega$. Define $\theta: \mathbb{Z}_{2} \times$ $[0,1] \rightarrow[0,1]$ by $\theta(0, \omega)=\omega$ and $\theta(1, \omega)=$ $1-\omega$. Also define $\varphi: \mathbb{Z}_{2} \times[0,1] \times \mathbb{R} \rightarrow \mathbb{R}$ by $\varphi(0, \omega) x=x$ and $\varphi(1, \omega) x=(1-2 \omega)+x$. Then $(\theta, \varphi)$ is RDS. Define $\zeta:[0,1] \rightarrow \mathbb{R}$ defined by $(\omega):=\omega^{2}$, then $\zeta \in \mathbb{R}_{\mathcal{B}}^{[0,1]}$. It is easy to see that $\zeta$ is a fixed point of $(\theta, \varphi)$.

Example1.7[9]: Let $\theta: \mathbb{R} \times \Omega \rightarrow \Omega$ be any non-trivial MDS and let $\eta: \Omega \rightarrow \mathbb{R}$ be any injective random variable. Define a cocycle $\varphi: \mathbb{R} \times \Omega \times \mathbb{R} \rightarrow \mathbb{R}$ over $\theta$ by

$\varphi(t, \omega) x:=x e^{-\eta(\omega)} e^{\eta(\theta(t) \omega)}$. Then $(\theta, \varphi)$ is RDS. This RDS has no random fixed point.

Definition 1.8[7] Let $v \in \mathbb{X}_{\mathcal{B}}^{\Omega}$ and $\gamma_{v}, \gamma_{v}^{+}$and $\gamma_{v}^{-}$be the mappings form $X$ in to $2^{X}$ defined as follows

(1) $\gamma_{v}(\omega)=\left\{\varphi\left(t, \theta_{-t} \omega\right) v\left(\theta_{-t} \omega\right): t \in \mathbb{R}\right\}$

(2) $\gamma_{v}^{+}(\omega)=\left\{\varphi\left(t, \theta_{-t} \omega\right) v\left(\theta_{-t} \omega\right): t \in \mathbb{R}^{+}\right\}$

(3) $\gamma_{v}^{-}(\omega)=\left\{\varphi\left(t, \theta_{-t} \omega\right) v\left(\theta_{-t} \omega\right): t \in \mathbb{R}^{-}\right\}$

For every $v \in X_{\mathcal{B}}^{\Omega}$, the sets $\gamma_{v}, \gamma_{v}^{+}$, and $\gamma_{v}^{-}$are respectively called the trajectory, the forward semi-trajectory and backward semi-trajectory.

\section{Main Results}

In this section the concept of random fixed point is stated and some new properties of such concept are studied. Also the concept of random periodic point for random dynamical systems is introduced and some new properties are given.

Lemma 2.1 If $v \in \mathbb{X}_{\mathcal{B}}^{\Omega}$ and

$\mathbb{P}\left\{\omega: \varphi(t, \omega) v(\omega)=v\left(\theta_{t} \omega\right)\right\}=1$ for some $t \in \mathbb{R}$, then $\mathbb{P}\left\{\omega: \varphi(n t, \omega) v(\omega)=v\left(\theta_{n t} \omega\right)\right\}=1$ for all integer $n$.

Proof. If $\mathbb{P}\left\{\omega: \varphi(t, \omega) v(\omega)=v\left(\theta_{t} \omega\right)\right\}=1$, for some $t \in \mathbb{R}$, then

$\mathbb{P}\left\{\omega: \varphi(t, \omega)^{-1} \circ \varphi(t, \omega) v(\omega)=\right.$ $\left.\varphi(t, \omega)^{-1} v\left(\theta_{t} \omega\right)\right\}=1$

Hence

$\mathbb{P}\left\{\omega: v(\omega)=\varphi\left(-t, \theta_{t} \omega\right) v\left(\theta_{t} \omega\right)\right\}=1$. Therefore we shall prove by induction the result for positive integers lone. If $n=1$, then

$$
\mathbb{P}\left\{\omega: \varphi(t, \omega) v(\omega)=v\left(\theta_{t} \omega\right)\right\}=1
$$

for some $t \in \mathbb{R}$.

Now, suppose that the statement is true for $n$. i.e., $\mathbb{P}\left\{\omega: \varphi(n t, \omega) v(\omega)=v\left(\theta_{n t} \omega\right)\right\}=1$,

for some $t \in \mathbb{R}$.

To show that this statement true for $n+1$. Set

$$
\widetilde{\Omega}:=\left\{\omega: \varphi(n t, \omega) v(\omega)=v\left(\theta_{n t} \omega\right)\right\} .
$$

For $\omega \in \widetilde{\Omega}$,

$$
\begin{gathered}
\varphi((n+1) t, \omega) v(\omega)=\varphi(n t+t, \omega) v(\omega) \\
=\varphi\left(n t, \theta_{t} \omega\right) \circ \varphi(t, \omega) v(\omega) \\
=\varphi\left(n t, \theta_{t} \omega\right) v\left(\theta_{t} \omega\right) \\
=\varphi\left(n t, \omega^{\prime}\right) v\left(\omega^{\prime}\right), \omega^{\prime}:=\theta_{t} \omega \in \widetilde{\Omega} \\
=v\left(\theta_{n t} \omega^{\prime}\right)=v\left(\theta_{n t} \theta_{t} \omega\right) \\
=v\left(\theta_{(n+1) t} \omega\right)
\end{gathered}
$$

Thus

$$
\mathbb{P}\left\{\omega: \varphi((n+1) t, \omega) v(\omega)=v\left(\theta_{(n+1) t} \omega\right)\right\}=1
$$

This prove the lemma. 
Theorem 2.2 Let $v \in \mathbb{X}_{\mathcal{B}}^{\Omega}$. Then the following are equivalent:

1. $v$ is random fixed point,

2. $\gamma_{v}(\omega)=\{v(\omega)\}$,

3. $\gamma_{v}^{+}(\omega)=\{v(\omega)\}$,

4. $\gamma_{v}^{-}(\omega)=\{v(\omega)\}$,

Proof.(1) $\Leftrightarrow(2)$ : Suppose(1) holds, then

$$
\begin{aligned}
& \gamma_{v}(\omega)=\left\{\varphi\left(t, \theta_{-t} \omega\right) v\left(\theta_{-t} \omega\right): t \in \mathbb{R}\right\} \\
= & \left\{\varphi\left(t, \omega^{\prime}\right) v\left(\omega^{\prime}\right): t \in \mathbb{R}\right\},
\end{aligned}
$$

where $\omega^{\prime}:=\theta_{-t} \omega$

$$
\begin{aligned}
& =\left\{v\left(\theta_{t} \omega^{\prime}\right): t \in \mathbb{R}\right\} \\
& =\left\{v\left(\theta_{t} \theta_{-t} \omega\right): t \in \mathbb{R}\right\}=\{v(\omega)\} .
\end{aligned}
$$

Conversely, suppose (2) holds, then

$\gamma_{v}(\omega)=\{v(\omega)\}$. But

$\gamma_{v}(\omega)=\left\{\varphi\left(t, \theta_{-t} \omega\right) v\left(\theta_{-t} \omega\right): t \in \mathbb{R}\right\}$, then

$\left\{\varphi\left(t, \theta_{-t} \omega\right) v\left(\theta_{-t} \omega\right): t \in \mathbb{R}\right\}=\{v(\omega)\} \quad$ That is $\forall t \in \mathbb{R}$ and $\forall \omega \in \Omega$,

$\varphi\left(t, \theta_{-t} \omega\right) v\left(\theta_{-t} \omega\right)=v(\omega)$. Thus $\forall t \in \mathbb{R}$

$\mathbb{P}\left\{\omega: \varphi\left(t, \theta_{-t} \omega\right) v\left(\theta_{-t} \omega\right)=v(\omega)\right\}=1$.

$\omega^{\prime}:=\theta_{-t} \omega$. Then for every $\in \mathbb{R}$,

$\varphi\left(t, \omega^{\prime}\right) v\left(\omega^{\prime}\right)=v\left(\theta_{t} \omega^{\prime}\right)$ and

$\mathbb{P}\left\{\omega^{\prime}: \varphi\left(t, \omega^{\prime}\right) v\left(\omega^{\prime}\right)=v\left(\theta_{t} \omega^{\prime}\right)\right\}=1$.

Consequently $v$ is an R.F.P.

$(2) \Leftrightarrow(3)$. Suppose (2) holds. Since $\gamma_{v}(\omega) \supset$ $\gamma_{v}^{+}(\omega) \neq \varnothing$, we conclude that $\gamma_{v}^{+}(\omega)=\{v(\omega)\}$. Conversely, suppose (3) holds. Then $\gamma_{v}^{+}(\omega)=$ $\{v(\omega)\}$. That is,

$\left\{\varphi\left(t, \theta_{-t} \omega\right) v\left(\theta_{-t} \omega\right): t \in \mathbb{R}^{+}\right\}=\{v(\omega)\}$. Then

$\left\{\varphi\left(s, \theta_{-s} \omega\right) v\left(\theta_{-s} \omega\right): s \in \mathbb{R}^{-}\right\}=\{v(\omega)\}, \quad$ where $s=-t$. Thus

$\left\{\varphi\left(t, \theta_{-t} \omega\right) v\left(\theta_{-t} \omega\right): t \in \mathbb{R}\right\}=\{v(\omega)\}$.

$(2) \Leftrightarrow$ (4). As in (2) $\Leftrightarrow$ (3).

This end the proof.

Theorem 2.3 Let $(\varphi, \theta)$ be an RDS with $\theta$ considered as a topological MDS. $\quad v \in \mathbb{X}_{\mathcal{B}}^{\Omega}$ is continuous, then the following are equivalent:

1. $v$ is random fixed point,
2. There is a sequence $\left\{t_{n}\right\}, t_{n}>0, t_{n} \rightarrow 0$ with

$$
\mathbb{P}\left\{\omega: \varphi\left(t_{n}, \omega\right) v(\omega)=v\left(\theta_{t_{n}} \omega\right)\right\}=1 .
$$

Proof To prove $(1) \Leftrightarrow(2)$. Assume (1). Since $v$ is random fixed point, then for all $\in \mathbb{R}$,

$$
\mathbb{P}\left\{\omega: \varphi(t, \omega) v(\omega)=v\left(\theta_{t} \omega\right)\right\}=1 .
$$

Thus we can say that there exists a real sequence $\left\{t_{n}\right\}, t_{n}>0, t_{n} \rightarrow 0$ with $\mathbb{P}\left\{\omega: \varphi\left(t_{n}, \omega\right) v(\omega)=v\left(\theta_{t_{n}} \omega\right)\right\}=1$.

Conversely, assume (2) holds, let $t \in \mathbb{R}$. If $t=k t_{n}$ for some integers $k$ and $n$, then by Lemma 2.1 we have

$\mathbb{P}\left\{\omega: \varphi\left(k t_{n}, \omega\right) v(\omega)=v\left(\theta_{k t_{n}} \omega\right)\right\}=1$.

If $t \neq k t_{n}$, then for every $n$, there exists $k_{n}$ such that $k_{n} t_{n}<t<\left(k_{n}+1\right) t_{n}$ and moreover for any $n$ there exist an integer $m>n$ with

$$
k_{n} t_{n}<k_{m} t_{m}<t<\left(k_{m}+1\right) t_{m}<\left(k_{n}+\right.
$$

1) $t_{n}$.

Thus clearly the so constructed sequence $\left\{k_{n} t_{n}\right\}$ has the property that $k_{n} t_{n} \rightarrow t$. Now since $\varphi(\cdot, \omega, v(\omega)): \mathbb{R} \rightarrow \mathbb{X}$ is continuous for every $\omega \in \Omega$, then $\varphi\left(k_{n} t_{n}, \omega\right) v(\omega) \rightarrow \varphi(t, \omega) v(\omega)$, for every $\omega \in \Omega$. Since

$$
\mathbb{P}\left\{\varphi\left(k_{n} t_{n}, \omega\right) v(\omega)=v\left(\theta_{k_{n} t_{n}} \omega\right)\right\}=1
$$

for every $n$ then for every $\omega \in \Omega$,

$v\left(\theta_{k_{n} t_{n}} \omega\right) \rightarrow \varphi(t, \omega) v(\omega)$.

Again, since $k_{n} t_{n} \rightarrow t$ and $\theta(\cdot, \omega): \mathbb{R} \rightarrow \Omega$ is continuous for every $\omega \in \Omega$, then $\theta_{k_{n} t_{n}} \omega \rightarrow$ $\theta_{t} \omega$, and since $v$ is continuous, then $v\left(\theta_{k_{n} t_{n}} \omega\right) \rightarrow v\left(\theta_{t} \omega\right)$ for every $\omega \in \Omega$. Thus $\varphi(t, \omega) v(\omega)=v\left(\theta_{t} \omega\right)$ for every $\omega \in \Omega$. This means that

$$
\mathbb{P}\left\{\omega: \varphi(t, \omega) v(\omega)=v\left(\theta_{t} \omega\right)\right\}=1
$$

for all $t \in \mathbb{R}$.

Note 2.4. The implication $(1) \Longrightarrow(2)$ is true when $\theta$ is any MDS. 
Definition 2.5 The set $\mathbb{X}_{\mathcal{B}}^{\Omega}$ is said to be distinguishable if for every $u, v \in \mathbb{X}_{\mathcal{B}}^{\Omega}$, there exist two random open sets $U$ and $V$ in $\mathbb{X}$ with $\mathfrak{D}:=$ $\{\omega: u(\omega) \neq v(\omega)\} \subseteq \Omega$ such that $\mathfrak{D} \neq \emptyset$, and for every $\omega \in \mathfrak{D}$ we have $u(\omega) \in U(\omega), \quad v(\omega) \in$ $V(\omega)$ and $U(\omega) \cap V(\omega)=\varnothing$. The set $\mathfrak{D}$ is said to be distinguish set.

Lemma 2.6 Let $\mathbb{X}_{\mathcal{B}}^{\Omega}$ is distinguishable with distinguish set $\mathfrak{D}$. If $v \in \mathbb{X}_{\mathcal{B}}^{\Omega}$ is not random fixed point, then there exist two random open sets $U$ and $V$ such that for every $\omega \in \mathfrak{D}$ with $v(\omega) \in$ $U(\omega)$ and $\varphi(t, \omega) v(\omega) \in V(\omega)$ we have $V(\omega)=$ $\varphi(t, \omega) U(\omega)$ and $V(\omega) \cap U(\omega)=\emptyset$.

Proof. Note that if $W$ is random open set in $\mathbb{X}$, then for every $\omega \in \Omega, \varphi(t, \omega) W(\omega)$ is random open set in $\mathbb{X}$, since $\varphi(t, \omega): \mathbb{X} \rightarrow \mathbb{X}$ is homeomorphism. Since $\mathbb{X}_{\mathcal{B}}^{\Omega}$ is distinguishable with distinguish set $\mathfrak{D}$, then there exist two random open sets $W_{1}$ and $W_{2}$ in $X$ such that for every $\omega \in \mathfrak{D} \quad$ we have $\quad v(\omega) \in W_{1}(\omega)$ and $\varphi(t, \omega) v(\omega) \in W_{2}(\omega)$ and $W_{1}(\omega) \cap W_{2}(\omega)=\varnothing$ for every $\omega \in \mathfrak{D}$. Since $v(\omega) \in W_{1}(\omega)$ for every $\in \mathfrak{D}$, then $\varphi(t, \omega) v(\omega) \in \varphi(t, \omega) W_{1}(\omega)$ for every $\omega \in \mathfrak{D}$. Set

$$
\begin{gathered}
V(\omega):= \\
\left\{\begin{array}{cc}
\varphi(t, \omega) W_{1}(\omega) \cap W_{2}(\omega), & \omega \in \mathfrak{D}^{c} \\
\emptyset, & \omega \in \mathfrak{D}^{c}
\end{array}\right.
\end{gathered}
$$

Then $\varphi(t, \omega) v(\omega) \in V(\omega)$, for every $\omega \in \mathfrak{D}$. Set $U(\omega):=\left\{\begin{array}{cc}\varphi(t, \omega)^{-1} V(\omega), & \omega \in \mathfrak{D} . \\ \emptyset, & \omega \in \mathfrak{D}^{c}\end{array}\right.$

Then $v(\omega) \in U(\omega)$, for every $\omega \in \mathfrak{D}$. Clearly that $V(\omega) \subseteq W_{2}(\omega)$ and $U(\omega) \subseteq W_{1}(\omega)$ for every $\omega \in \mathfrak{D}$. But $W_{1}(\omega) \cap W_{2}(\omega)=\varnothing$ for every $\omega \in \mathfrak{D}$, this implies that $U(\omega) \cap V(\omega)=\emptyset$, for every $\omega \in \mathfrak{D}$.
Theorem 2.7 Let $\mathbb{X}_{\mathcal{B}}^{\Omega}$ is distinguishable with distinguish set $\mathfrak{D}$. Then $v \in \mathbb{X}_{\mathcal{B}}^{\Omega}$ is random fixed point if and only if every random neighborhood of $v$, contains semi-trajectory for all $\omega \in \mathfrak{D}$.

Proof Suppose that $v \in \mathbb{X}_{\mathcal{B}}^{\Omega}$ is random fixed point, then $\gamma_{v}(\omega)=\{v(\omega)\}$ so that $\gamma_{v}(\omega)$ contained in every random neighborhood of $v$. Conversely, suppose that every random neighborhood of $v$ contains semi-trajectory. Assume contrary that $v \in \mathbb{X}_{\mathcal{B}}^{\Omega}$ is not random fixed point, then there exists $t \in \mathbb{R}^{+}$, such that for every $\widetilde{\Omega} \subseteq \Omega$ with $\mathbb{P}(\widetilde{\Omega})=$ $1, \varphi(t, \omega) v(\omega) \neq v\left(\theta_{t} \omega\right)$, for some $\omega \in \widetilde{\Omega}$. Вy Lemma 2.6 there exist two random open sets $U$ and $V$ such that for every $\omega \in \mathfrak{D}$ with $v(\omega) \in$ $U(\omega)$ and $\varphi(t, \omega) v(\omega) \in V(\omega)$ we have $V(\omega)=$ $\varphi(t, \omega) U(\omega)$ and $V(\omega) \cap U(\omega)=\emptyset$. Since for each $u(\omega) \in U(\omega), \quad \omega \in \mathfrak{D} \quad$ we have $\varphi(t, \omega) u(\omega) \in V(\omega), \quad \omega \in \mathfrak{D}, \quad$ then $\varphi(t, \omega) u(\omega) \notin U(\omega), \quad \omega \in \mathfrak{D}$. But this is a contradiction.

Theorem 2.8 Let $\mathbb{X}_{\mathcal{B}}^{\Omega}$ is distinguishable with distinguish invariant set $\mathfrak{D}$.If $u, v \in X_{\mathcal{B}}^{\Omega}$ and $d\left(\varphi\left(t, \theta_{-t} \omega\right) v\left(\theta_{-t} \omega\right), u(\omega)\right) \rightarrow 0, \quad$ for every $\omega \in \Omega$ as $t \rightarrow+\infty$ ( or $t \rightarrow-\infty$ ). Then $u$ is random fixed point.

Proof. Let $U$ be a random neighborhood of $u$. Since $\quad d\left(\varphi\left(t, \theta_{-t} \omega\right) v\left(\theta_{-t} \omega\right), u(\omega)\right) \rightarrow 0, \quad$ for every $\omega \in \Omega$ as $t \rightarrow+\infty$, there exists $T \geq 0$ such that

$$
\varphi\left(t, \theta_{-t} \omega\right) v\left(\theta_{-t} \omega\right) \in U(\omega)
$$

for every $\omega \in \Omega$, for all $t \geq T$. Hence for all $\omega \in \mathfrak{D}$ we have $\left\{\varphi(s, \omega) \varphi\left(t, \theta_{-t} \omega\right) v\left(\theta_{-t} \omega\right): s \in \mathbb{R}^{+}\right\} \in U(\omega)$.

Then for all $\omega \in \mathfrak{D}$, $\left\{\varphi\left(s+t, \theta_{-t} \omega\right) v\left(\theta_{-t} \omega\right): s \in \mathbb{R}^{+}\right\} \in U(\omega)$. Or for all $\omega \in \mathfrak{D}$,

$\left\{\varphi\left(r, \omega^{\prime}\right) v\left(\omega^{\prime}\right): r \in \mathbb{R}^{+}\right\} \in U\left(\theta_{t} \omega^{\prime}\right)$, 
where $r=s+t$ and $\omega^{\prime}:=\theta_{-t} \omega$. That is $U$ contains semi-trajectory, consequently, by Theorem $2.7 u$ is R.F.P.

Proposition 2.9 Let $v \in \mathbb{X}_{\mathcal{B}}^{\Omega}$ be a random fixed point. If $u \in \mathbb{X}_{\mathcal{B}}^{\Omega}$ with $\mathbb{P}\{\omega: v(\omega) \neq u(\omega)\}=1$, then

$\mathbb{P}\left\{\omega: \varphi(t, \omega) u(\omega)=v\left(\theta_{t} \omega\right)\right\} \neq 1$.

Proof. Suppose that $v \in \mathbb{X}_{\mathcal{B}}^{\Omega}$ is a random fixed point. Let $u \in \mathbb{X}_{\mathcal{B}}^{\Omega}$ with

$$
\mathbb{P}\{\omega: v(\omega) \neq u(\omega)\}=1 .
$$

Assume contrary that

$$
\mathbb{P}\left\{\omega: \varphi(t, \omega) u(\omega)=v\left(\theta_{t} \omega\right)\right\}=1 .
$$

Then $\mathbb{P}\{\omega: \varphi(t, \omega) u(\omega)=\varphi(t, \omega) v(\omega)\}=1$

Since $\varphi(t, \omega)$ is bijective, then

$$
\mathbb{P}\{\omega: u(\omega)=v(\omega)\}=1 .
$$

So $\mathbb{P}\{\omega: u(\omega) \neq v(\omega)\}=0$.

Which is a contradiction.

In [9] I.J.Kadhim introduce the concept of random periodic point for random dynamical system. Here we define this concept in another manner which more suitable with our work .

Definition 2.10 A random variable $v \in \mathbb{X}_{\mathcal{B}}^{\Omega}$ is said to be random periodic point of a $\operatorname{RDS}(\theta, \varphi)$ if there exists $\tau \neq 0$ such that

$$
\mathbb{P}\left\{\omega: \varphi(\tau, \omega) v(\omega)=v\left(\theta_{\tau} \omega\right)\right\}=1 \text {. The number }
$$
$\tau$ is called the period of $v$.

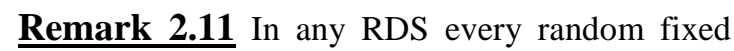
point is random periodic point.

Proposition 2.12 A random variable $v \in \mathbb{X}_{\mathcal{B}}^{\Omega}$ is random periodic point if and only if there exists $\tau \neq 0$ such that for every $t \in \mathbb{R}, \mathbb{P}\{\omega: \varphi(t+$ $\left.\tau, \omega) v(\omega)=\varphi\left(t, \theta_{\tau} \omega\right) v\left(\theta_{\tau} \omega\right)\right\}=1$

Proof. Suppose that $v \in \mathbb{X}_{\mathcal{B}}^{\Omega}$ is random periodic point. Then there exists $\tau \neq 0$ such that

$\mathbb{P}\left\{\omega: \varphi(\tau, \omega) v(\omega)=v\left(\theta_{\tau} \omega\right)\right\}=1$.

If and only if
$\mathbb{P}\left\{\omega: \varphi\left(t, \theta_{\tau} \omega\right) \varphi(\tau, \omega) v(\omega)=\right.$

$\left.\varphi\left(t, \theta_{\tau} \omega\right) v\left(\theta_{\tau} \omega\right)\right\}=1, \forall t \in \mathbb{R}$

If and only if

$\mathbb{P}\left\{\omega: \varphi(t+\tau, \omega) v(\omega)=\varphi\left(t, \theta_{\tau} \omega\right) v\left(\theta_{\tau} \omega\right)\right\}=1$,

$\forall t \in \mathbb{R}$

This complete the proof.

Theorem 2.13 Let $(\theta, \varphi)$ be an RDS with $\theta$ be a stable TMDS and let $v \in \mathbb{X}_{\mathcal{B}}^{\Omega}$ be a random periodic point and continuous but not R.F.P. Then there exists $T>0$ such that $T$ is the smallest positive period of $v$.Further, if $\tau$ is any other positive period of $v$, then $\tau=n T$ for some integer $n$.

Proof. Consider the set

$S:=\{t>0: t$ the period of $v\}$.

If $T \neq 0$ period of $v$, then

$\mathbb{P}\left\{\omega: \varphi(T, \omega) v(\omega)=v\left(\theta_{T} \omega\right)\right\}=1$

Let $\omega^{\prime}:=\theta_{T} \omega$, then $\omega=\theta_{-T} \omega^{\prime}$. Since

$\mathbb{P}\left\{\omega: \varphi(T, \omega) v(\omega)=v\left(\theta_{T} \omega\right)\right\} \equiv$ $\mathbb{P}\left\{\omega^{\prime}: \varphi\left(T, \theta_{-T} \omega^{\prime}\right) v\left(\theta_{-T} \omega^{\prime}\right)=v\left(\omega^{\prime}\right)\right\}$,

then

$\mathbb{P}\left\{\omega^{\prime}: \varphi\left(T, \theta_{-T} \omega^{\prime}\right) v\left(\theta_{-T} \omega^{\prime}\right)=v\left(\omega^{\prime}\right)\right\}=1$.

Now, set

$\widetilde{\Omega}:=\left\{\omega^{\prime}: \varphi\left(T, \theta_{-T} \omega^{\prime}\right) v\left(\theta_{-T} \omega^{\prime}\right)=v\left(\omega^{\prime}\right)\right\}$.

show that

$$
\begin{aligned}
& \varphi\left(-T, \omega^{\prime}\right) v\left(\omega^{\prime}\right)=v\left(\theta_{-T} \omega^{\prime}\right), \forall \omega^{\prime} \in \widetilde{\Omega}: \\
& \varphi\left(-T, \omega^{\prime}\right) v\left(\omega^{\prime}\right) \\
& \quad=\varphi\left(-T, \omega^{\prime}\right) \varphi\left(T, \theta_{-T} \omega^{\prime}\right) v\left(\theta_{-T} \omega^{\prime}\right) \\
& =\varphi\left(-T+T, \omega^{\prime}\right) v\left(\theta_{-T} \omega^{\prime}\right)=v\left(\theta_{-T} \omega^{\prime}\right) .
\end{aligned}
$$

Hence

$\mathbb{P}\left\{\omega^{\prime}: \varphi\left(-T, \omega^{\prime}\right) v\left(\omega^{\prime}\right)=v\left(\theta_{-T} \omega^{\prime}\right)\right\}=1$. Since either $T$ or $-T$ is positive, then the set $S$ is nonempty. Now, set $\inf S=T$. We calem that $T>$ 0 . Indeed $T \geq 0$, and if $T=0$, then there exists a sequence $\left\{t_{n}\right\}$ in $S$ with $t_{n} \rightarrow 0$. Since

$\mathbb{P}\left\{\omega: \varphi\left(t_{n}, \omega\right) v(\omega)=v\left(\theta_{t_{n}} \omega\right)\right\}=1$ 
Sundus .T/Ihsan .J

for each $n$, then by Theorem $2.3 v$ is random fixed point which contradicts our hypothesis. Thus $T>0$. Since $\inf S=T$, then there is a sequence $\left\{t_{n}\right\}$ in $S$ with $t_{n} \rightarrow T$. Since $\varphi(\cdot, \omega): \mathbb{R} \rightarrow \mathbb{X}$ is continuous for every $\omega \in \Omega$, then for every $\omega \in \Omega$, $\varphi\left(t_{n}, \omega\right) \rightarrow \varphi(T, \omega)$.So for every $\omega \in \Omega$,

$$
\varphi\left(t_{n}, \omega\right) v(\omega) \rightarrow \varphi(T, \omega) v(\omega)
$$

Since $\theta(\cdot, \omega): \mathbb{R} \rightarrow \Omega$ is continous for every $\omega \in \Omega$, then $\theta_{t_{n}} \omega \rightarrow \theta_{T} \omega$ for every $\omega \in \Omega$. Again, since $v \in \mathbb{X}_{\mathcal{B}}^{\Omega}$ is continuous, then $v\left(\theta_{t_{n}} \omega\right) \rightarrow v\left(\theta_{T} \omega\right)$ for every $\quad \omega \in \Omega$. But $\mathbb{P}\left\{\omega: \varphi\left(t_{n}, \omega\right) v(\omega)=\right.$ $\left.v\left(\theta_{t_{n}} \omega\right)\right\}=1$,

i.e., there exists a full measure subset $\widetilde{\Omega}$ of $\Omega$ such that $\varphi\left(t_{n}, \omega\right) v(\omega)=v\left(\theta_{t_{n}} \omega\right)$ for every $\omega \in \widetilde{\Omega}$. Hence $\forall \omega \in \widetilde{\Omega}$,

$v\left(\theta_{t_{n}} \omega\right) \rightarrow \varphi(T, \omega) v(\omega),$.

On the other hand, $v\left(\theta_{t_{n}} \omega\right) \rightarrow v\left(\theta_{T} \omega\right)$, for every $\omega \in \widetilde{\Omega}$. Since $\mathbb{X}$ is metric space, then from the uniqenss of the limit we have $\varphi(T, \omega) v(\omega)=$ $v\left(\theta_{T} \omega\right) \forall \omega \in \widetilde{\Omega}$.

That is, $\mathbb{P}\left\{\omega: \varphi(T, \omega) v(\omega)=v\left(\theta_{T} \omega\right)\right\}=1$. It follows that $T \in S$. By definition of $T$ it is also the smallest positive period of $v$. Finally, let $t \in \mathbb{R}$ be a positive periodic. If $t \neq n T$, for any integer, then there is an integer $n$ with $n T<t<(n+1) T$. Then by Lemma 2.1, we have

$\mathbb{P}\left\{\omega: \varphi(n T, \omega) v(\omega)=v\left(\theta_{n T} \omega\right)\right\}=1$.

Since the TMDS $\theta$ is satble, then

$$
\mathbb{P}\left\{\omega: \theta_{t} \omega=\theta_{n T} \omega\right\}=1 \text {. }
$$

Therefore by Lemma 2.1 we have

$\mathbb{P}\{\omega: \varphi(n T, \omega) v(\omega)=\varphi(t, \omega) v(\omega)\}=1$. So

$\mathbb{P}\left\{\omega: v(\omega)=\varphi\left(-n T, \theta_{n T} \omega\right) \circ \varphi(t, \omega) v(\omega)\right\}=1$.

Thus

$$
\begin{aligned}
& \varphi(n T, \omega) v\left(\theta_{t-n T} \omega\right)=\varphi(t, \omega) v(\omega) \\
& v\left(\theta_{t-n T} \omega\right)=\varphi\left(-n T, \theta_{t-n T} \omega\right) \varphi(t, \omega) v(\omega) \\
& \varphi(\tau, \omega) v(\omega)=v\left(\theta_{\tau} \omega\right)
\end{aligned}
$$

where $\tau:=t-n T$. Then $\tau$ satisfy (2.1). Since $0<\tau<T$, we get a contradiction to the fact that $T$ was the smallest positive period of period of $v$. This complete the prove.

In the following we need to show that the set of random periodic point for random dynamical system (under certain conditions) is $\mathbb{P}$-closed. To this end the following notations are introduced.

Definition 2.14 Let $(\Omega, \mathbb{F}, \mathbb{P})$ be a probability space with $\Omega$ considered as a topological space and $\mathbb{X}$ be any metric space. A sequence $\left\{v_{n}\right\}$ in $\mathbb{X}_{\mathcal{B}}^{\Omega}$ is said to be $\mathbb{P}$-uniform converge in $\mathbb{X}_{\mathcal{B}}^{\Omega}$ if there exist $v \in \mathbb{X}_{\mathcal{B}}^{\Omega}$ and $\widetilde{\Omega} \subseteq \Omega$ such that $v_{n}(\omega)$ converge uniformly (shortly u.c.) to $v(\omega)$ for every $\omega \in \widetilde{\Omega}$. That is for every $\varepsilon>0$, there is a positive integer $n_{0}$ such that

$d\left(v_{n}(\omega), v(\omega)\right)<\varepsilon$, for every $\omega \in \widetilde{\Omega}$ and for every $n>n_{0}$.

Definition 2.15 Let $(\Omega, \mathbb{F}, \mathbb{P})$ be a probability space with $\Omega$ considered as a topological space and $\mathbb{X}$ be any topological space. A subset of $\mathbb{X}_{\mathcal{B}}^{\Omega}$ is said to be $\mathbb{P}$-closed if any sequence $\left\{v_{n}\right\}$ in $\mathbb{X}_{\mathcal{B}}^{\Omega}$ is $\mathbb{P}$-uniform converge in $\mathbb{X}_{\mathcal{B}}^{\Omega}$.

Lemma 2.16 Let $(\theta, \varphi)$ be an $\operatorname{RDS}$ with $\theta$ is be a TMDS. If $\left\{v_{n}\right\}$ be a sequence of continous random periodic point in $\mathbb{X}_{\mathcal{B}}^{\Omega}$ with positive periodic $T_{n} \rightarrow 0$, and $\mathbb{P}$-uniform converge in $\mathbb{X}_{\mathcal{B}}^{\Omega}$, then $v$ is random fixed point.

Proof. For a given $t \in \mathbb{R}$, there are integers $k_{n}$ such that

$k_{n} T_{n} \leq t<k_{n} T_{n}+T_{n}$.

Since $T_{n} \rightarrow 0$, we have $k_{n} T_{n} \rightarrow t$. Since $v_{n}$ is $\mathbb{P}$-uniform converge to $v$, then $\mathbb{P}\left\{\omega: v_{n}(\omega)\right.$ u. c. to $\left.v(\omega)\right\}=1$. Let $\Omega_{1}:=\left\{\omega: v_{n}(\omega)\right.$ u.c. to $\left.v(\omega)\right\}$, then $\quad \mathbb{P}\left(\Omega_{1}\right)=1$ But $\varphi(\cdot, \omega, \cdot): \mathbb{R} \times \mathbb{X} \rightarrow \mathbb{X}$ is continuous for every $\omega$, then for every $\omega \in \Omega_{1}$, 
$\varphi\left(k_{n} T_{n}, \omega\right) v_{n}(\omega) \rightarrow \varphi(t, \omega) v(\omega)$

Since $\theta(\cdot, \omega): \mathbb{R} \rightarrow \Omega$ is continuous for every $\omega$, then $\theta_{k_{n} T_{n}} \omega \rightarrow \theta_{t} \omega$ for every $\omega$. Therefore $v_{n}\left(\theta_{k_{n} T_{n}} \omega\right) \rightarrow v_{n}\left(\theta_{t} \omega\right)$ for every $\omega$. Since $v_{n}\left(\theta_{t} \omega\right) \rightarrow v\left(\theta_{t} \omega\right), \quad$ for every $\in \Omega_{1}$, then $v_{n}\left(\theta_{k_{n} T_{n}} \omega\right) \rightarrow v\left(\theta_{t} \omega\right)$ for every $\omega \in \Omega_{1}$. Since $v_{n}$ is random periodic point for every $n$, then

$$
\mathbb{P}\left\{\omega: \varphi\left(k_{n} T_{n}, \omega\right) v_{n}(\omega)=v_{n}\left(\theta_{k_{n} T_{n}} \omega\right)\right\}=1
$$

Set

$\Omega_{2}:=\left\{\omega: \varphi\left(k_{n} T_{n}, \omega\right) v_{n}(\omega)=v_{n}\left(\theta_{k_{n} T_{n}} \omega\right)\right\}$. Then $\mathbb{P}\left(\Omega_{2}\right)=1$. So

$\varphi\left(k_{n} T_{n}, \omega\right) v_{n}(\omega)=v_{n}\left(\theta_{k_{n} T_{n}} \omega\right), \quad$ for every $\omega \in \Omega_{2}$.

$\varphi\left(k_{n} T_{n}, \omega\right) v_{n}(\omega) \rightarrow \varphi(t, \omega) v(\omega)$,

for every $\omega \in \Omega_{1}$. Then,

$v_{n}\left(\theta_{k_{n} T_{n}} \omega\right) \rightarrow \varphi(t, \omega) v(\omega)$, for every $\omega \in \Omega_{2} \cap$ $\Omega_{1}$

Since $v_{n}\left(\theta_{k_{n} T_{n}} \omega\right) \rightarrow v\left(\theta_{t} \omega\right)$ for every $\omega \in \Omega_{1}$,

it follwos that

$\varphi(t, \omega) v(\omega)=v\left(\theta_{t} \omega\right)$, for every $\omega \in \Omega_{2} \cap \Omega_{1}$.

Since $\mathbb{P}\left(\Omega_{2} \cap \Omega_{1}\right)=1$, then $v$ is random fixed point.

Theorem 2.17 Let $(\theta, \varphi)$ be an $\operatorname{RDS}$ with $\theta$ is be a TMDS .Given any $\alpha>0$, the set of all $v$ such that $v$ is (continuous) random periodic point with positive period $T \leq \alpha$ is $\mathbb{P}$-closed.

Proof. Let $P$ be a set of all random periodic point with positive period $T \leq \alpha$. Suppose that $\left\{v_{n}\right\}$ be $\mathbb{P}$-uniform converge sequence in $P$. Then for every $n, v_{n}$ is random periodic point with periods $T_{n} \leq \alpha$ and $v_{n}$ then

$$
\mathbb{P}:=\left\{\omega: v_{n}(\omega) \rightarrow v(\omega)\right\}=1 \text {. }
$$

Set

$\Omega_{1}:=\left\{\omega: v_{n}(\omega) \rightarrow v(\omega)\right\}$,

then $\mathbb{P}\left(\Omega_{1}\right)=1$. Since

$\mathbb{P}\left\{\omega: \varphi\left(T_{n}, \omega\right) v_{n}(\omega)=v_{n}\left(\theta_{T_{n}} \omega\right)\right\}=1, \quad$ then $\mathbb{P}\left(\Omega_{2}\right)=1$, where
$\Omega_{2}:=\left\{\omega: \varphi\left(T_{n}, \omega\right) v_{n}(\omega)=v_{n}\left(\theta_{T_{n}} \omega\right)\right\}$. Also for every $\in \Omega_{1}$,

$\mathbb{P}\left\{\omega: v_{n}\left(\theta_{T_{n}} \omega\right) \rightarrow v\left(\theta_{T_{n}} \omega\right)\right\}=1$.

Since $0 \leq T_{n} \leq \alpha$, either $T_{n} \rightarrow 0$ in which case $v$ is random fixed point by Lemma 2.15 and hence random periodic, or there is a subsequence $T_{n_{k}} \rightarrow \tau$, $0<\tau \leq \alpha$, then by the continuity axiom for every $\omega \in \Omega_{1}$

$\varphi\left(T_{n_{k}}, \omega\right) v_{n_{k}}(\omega) \rightarrow \varphi(\tau, \omega) v(\omega)$.

and also for every $\omega \in \Omega_{2}$,

$$
\varphi\left(T_{n_{k}}, \omega\right) v_{n_{k}}(\omega) \rightarrow v\left(\theta_{T_{n_{k}}} \omega\right)
$$

Since $\theta$ is continuous, then $\theta_{T_{n_{k}}} \omega \rightarrow \theta_{\tau} \omega$ for every $\omega$. Also we have $v$ is continuous for every $\omega \in \Omega_{1}$, then $\quad v\left(\theta_{T_{n_{k}}} \omega\right) \rightarrow v\left(\theta_{\tau} \omega\right), \quad$ for every $\omega \in$ $\Omega_{1}$. Therefore $\left.\varphi\left(T_{n_{k}}, \omega\right) v_{n_{k}}(\omega)\right) \rightarrow v\left(\theta_{\tau} \omega\right)$. for every $\omega \in \Omega_{2} \cap \Omega_{1}$. Consequently $\varphi(\tau, \omega) v(\omega)=v\left(\theta_{\tau} \omega\right)$ for every $\omega \in \Omega_{2} \cap \Omega_{1}$. Since $\mathbb{P}\left(\Omega_{2} \cap \Omega_{1}\right)=1$, then $v$ is random periodic point.

\section{References}

[1] L. Arnold and I.D. Chueshov,"Order Preserving Random Dynamical Systems: Equilibria, attractor , applications", Dynamics and Stability of Systems,13(1998), 265-280 .

[2] Gunter Ochs and Valery. Oseledets," Topological Fixed Point Theorems Do Not Hold for Random Dynamical Systems" Journal of Dynamics and Differential Equations, Vol. II, No. 4, 1999.

[3] H.E. Kunze D. La Torreb and E.R. Vrscay ," Random fixed point equations and inverse problems using "collage method" for contraction mappings",J. Math. Anal. Appl. 334 (2007) 1116-1129.

[4] Chuanxi Zhu and Chunfang Chen," Calculations of random fixed point index, J. Math. Anal. Appl. 339 (2008) 839-844. 
[5] Ismat Beg and Mujahid Abbas," Random fixed point theorems for a random operator on an unbounded subset of a Banach space",Applied Mathematics Letters 21 (2008) 1001-1004.

[6] L. Arnold ,Random dynamical systems, Springer, Berlin (Corrected 2nd printing)(2003).

[7] I. Chueshov " Monotone Random Systems Theory and Applications" Springer- Verlag Berlin Heidelberg Germany (2002).
[8] H. Crauel and M. Gundlach, " Stochastic Dynamics" Springer-Verlag New York, Inc. (1999).

[9] I.J. Kadhim," Some Results on Random Dynamical Systems" P.h.D thesis, University of Al-Mustansiriyah, Iraq. (2016).

\title{
بعض خواص النقاط الصامدة العثوائية و النقاط الدورية العثوية للنظم الديناميكية العثوائية
}

\author{
سندس طالب محسن أحسان جبار كاظم \\ جامعة القادية / كلية علوم الحاسوب وتكنولوجيا المعلومات / قسم الرياضيات
}

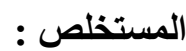

الهدف الرئيسي من هذا البحث هو اعطاء بعض الخواص النقاط الصامدة العشوائية للنظم الديناميكية العشوائية

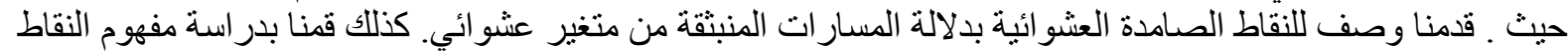

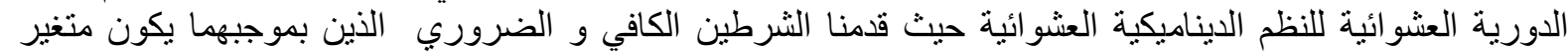
عشو ائي نقطة دورية عشوائبة كما وقد برهنا ان مجموعة كل النقاط الدورية (المستمرة) تحت شروط معينة، تكون 